Artículo Original. Enero-Abril 2017; 7(1):26-35. Recibido: 03/10/2016 Aceptado: 23/02/2017.

http://dx.doi.org/10.21929/abavet2017.71.2

\title{
Evaluación de semilla de guayaba (Psidium guajava L.) como alternativa en la nutrición ruminal
}

\section{Evaluation of seed of guava (Psidium guajava $L$.$) as an alternative in ruminal$ nutrition}

\author{
Silva-Vega Mónica ${ }^{1 *}$ msilva58@hotmail.com, Bañuelos-Valenzuela Rómulo ${ }^{1 * *}$ \\ apozolero@hotmail.com, Muro-Reyes Albertoa amurey@hotmail.com, Esparza-Ibarra Edgar ${ }^{2}$ \\ edgarzac@gmail.com, Delgadillo-Ruiz Lucía² delgadillolucia@gmail.com \\ 1 Unidad Académica de Medicina Veterinaria y Zootecnia. Universidad Autónoma de Zacatecas. México. \\ 2Unidad Académica de Ciencias Biológicas. Universidad Autónoma de Zacatecas. México. Autor \\ responsable*: Silva-Vega Mónica. Autor de correspondencia**: Bañuelos-Valenzuela Rómulo. Carretera \\ panamericana Zacatecas-Fresnillo, km 31.5 Calera de Víctor Rosales, Zacatecas, C. P. 98500. México.
}

\section{ABSTRACT}

The nutritional contents of guava flour and germinated guava seeds were evaluated. A proximal analysis and in vitro digestibility were performed. There were significant differences between the flour and germinated guava seeds $(p<0.01)$ for a percentage of ash, crude protein, crude fiber and neutral detergent fiber. In the in vitro digestibility, the highest gas production was given by germination, with $40 \mathrm{ml}$ of gas per $200 \mathrm{mg}$ of dry matter. The highest production of volatile fatty acids after in vitro digestibility (48 h) occurred in the germination of guava seed. Basing on these results, it is concluded that the flour and germinated guava seeds have the possibility of being used in the diet of ruminants.

Keywords: Guava, proximal analysis, digestibility.

\section{RESUMEN}

Para evaluar los contenidos nutricionales de la semilla de guayaba se emplearon harina y germinado de la semilla. Se realizó un análisis proximal y digestibilidad in vitro. Existieron diferencias significativas entre la harina y germinado de semilla de guayaba $(p<0.01)$ para porcentaje de ceniza, proteína cruda, fibra cruda y fibra detergente neutra. En la digestibilidad in vitro la mayor producción de gas se dio en germinado, con $40 \mathrm{ml}$ de gas por cada $200 \mathrm{mg}$ de materia seca. La mayor producción de ácidos grasos volátiles después de la digestibilidad in vitro (48 h) ocurrió en el germinado de semilla de guayaba. Se concluye que la harina y germinado de semilla de guayaba tienen posibilidad de ser utilizadas en la dieta de rumiantes.

Palabras claves: Guayaba, análisis proximal, digestibilidad. 


\section{INTRODUCCIÓN}

México ocupa el quinto lugar mundial en la producción de guayaba con 298,062 toneladas, que representan el $4.4 \%$ de la oferta internacional, y es superado en la producción por países como Pakistán, China e India (SAGARPA, 2015). La producción nacional se registra en 22 estados con 22,576 hectáreas sembradas, de las cuales el $89 \%$ (22,246 ha) se cosechan en los estados de Michoacán, Aguascalientes y Zacatecas (SIAP, 2012).

El guayabo es un árbol pequeño (con una altura promedio de 2 a 7 metros), comparado con árboles como el de manzano (12 m), naranjo (13 m), o mango (30 m). Las hojas del guayabo son opuestas, de forma oblonga, con venas prominentes de abajo. Las flores son de color blanco y de $2.5 \mathrm{~cm}$ de diámetro; los frutos son de forma ovoides y vuelta a forma de pera, con cáscara delgada, con semillas incrustadas en una pulpa firme; o sin cáscara gruesa con pocas semillas (Rishika y Sharma, 2012). Las semillas representan $12 \mathrm{~g} / 100 \mathrm{~g}$ de peso de la fruta y la mayoría de las veces se descartan; sin embargo, este material vegetal tiene un alto valor nutricional, proteína cruda de $9.7 \%$ y una digestibilidad mayor que la de soya (Bernardino et al., 2001), que puede ser empleado como alimento de rumiantes.

La longevidad de la semilla de guayaba está determinada por su constitución genética, las características fisiológicas y por daños previos o durante el almacenamiento (Willan, 1991). Cuando la siembra se realiza en vermiculita, papel filtro o toallas de papel a temperaturas de 15 a $35^{\circ} \mathrm{C}$, no existen diferencias en los porcentajes de germinación en el género Psidium guajava (Pereira y Andrade, 1994). Otra alternativa que favorece la germinación y emergencia de la plántula criptocotilar entre 15 y 23 días es el remojo durante 24 horas (Meza y Bautista, 2007).

Por el alto contenido de proteína vegetal de la semilla y la relativa facilidad en la germinación, la semilla de guayaba se ha considerado como una alternativa de alimento para rumiantes. La fibra contribuye al mantenimiento del funcionamiento y $\mathrm{pH}$ ruminal; la secreción salival dependiente de la masticación y la rumia, cuyas funciones dependen de la composición, la degradabilidad y la forma de presentación de la fibra, a pesar de limitar el contenido energético de las raciones y el potencial de ingestión (Mertens, 1987).

En rumiantes, los ácidos grasos volátiles (AGV), son los productos finales de la fermentación de la materia orgánica del alimento y representan la principal fuente de energía (Sutton, 1980); donde la producción y proporción de los AGV acético, propiónico y butírico varía en función de: la dieta administrada, la relación concentrado-forraje, el tipo de procesamiento físico al que fue sometido el alimento (Kauffman, 1976), la degradabilidad de la fibra (Beharka y Nagaraja, 1998) y la digestibilidad (France et al., 
1991). Debido a lo anterior, el objetivo fue evaluar el contenido nutricional de la harina de semilla y germinado de guayaba para su uso en la alimentación de rumiantes.

\section{MATERIAL Y MÉTODOS}

Los frutos de guayaba fueron colectados en el municipio de Jalpa, Zacatecas (Latitud $21^{\circ} 38^{\prime} 23^{\prime \prime}$ Norte y Longitud $102^{\circ} 58^{\prime} 47^{\prime \prime}$ Oeste), cuyas condiciones climáticas son de semiseco a semicálido. Se recolectaron guayabas en diversos estados de maduración; en época de mayor cosecha que comprendió el periodo de los meses de septiembre de 2012 a febrero del 2013. El fruto se clasificó por estado de maduración, según lo establecido por Del Pilar et al. (2007).

1. Verde.- Fruto con cáscara rígida, de coloración verde.

2. Maduro.- Cáscara rígida con coloración amarilla.

3. Postmaduro.- Cáscara que carece de rigidez con coloración amarillo-rojizo.

Las semillas de guayaba fueron separadas de la pulpa, se pesaron e identificaron por unidad de fruto y estado de maduración. Una vez secas fueron asignadas al azar para su germinación. Una primera germinación se realizó en recipientes de plástico con capacidad de un litro que contenían toallas de papel y gasas, donde se colocaron 100 semillas y se cuantificó el porcentaje de germinación. La segunda consistió en colocar cinco semillas en pozos de placas de germinación de 200 pozos de unicel, a una profundidad de un centímetro con sustrato inerte de la marca comercial $L A M B E R T \angle M \circledast$, obtenido de Turba Sphagnum canadiense.

Las semillas se regaron diariamente durante cinco semanas. Al finalizar la germinación se observó el brote de la plántula y fueron medidas desde las hojas hasta la raíz. En la segunda germinación fueron recolectadas las plántulas, las cuales fueron secadas y molidas para realizar el análisis proximal. Para la obtención de la harina, las semillas de guayaba se molieron en un molino de alimentos marca comercial.

\section{Análisis proximal}

El análisis consistió en la determinación de: cenizas, extracto etéreo, fibra cruda ( $F C)$, fibra detergente neutro (FDN), fibra detergente ácido (FDA), proteína y materia seca (MS). La cuantificación de cenizas se realizó según la metodología descrita por la (AOAC, 1995). Las muestras se analizaron para fibras por triplicado en un digestor ANKOM de 65 rpm de agitación (ANKOM 200, Tecnology, USA). Las proteínas se determinaron por el método de Dumas descrito por primera vez por Jean-Baptiste Dumas en 1826, que consiste en la conversión de los gases de combustión. Los resultados se expresaron como porcentaje de proteína. 


\section{Degradabilidad in vitro por producción de gas}

Alimentación de los ovinos

Para la producción de gas in vitro se utilizó fluido ruminal de 2 ovinos de pelo, canulados y alimentados con una dieta que contenía $83 \%$ de heno y $17 \%$ de concentrado (NCR, 2007). El alimento se proporcionó diariamente a las 08:00 y 16:00 horas con acceso libre al agua. Se alimentó a los ovinos por 30 días antes de la extracción del fluido ruminal, como tiempo de adaptación a la ración.

Producción de gas in vitro.

La fermentación se midió a través de la producción de gases, principalmente de metano y bióxido de carbono, los cuales generan una cinética de digestión, midiendo la fermentación del alimento en lugar de su desaparición, técnica descrita por Van Soest (1994). Para lo cual se utilizaron unidades de fermentación (UF) de $120 \mathrm{~mL}$ para cada muestra. A las UF se le agregaron $700 \pm 10 \mathrm{mg}$ de materia seca (MS) de cada una de las muestras; además se adicionaron $90 \mathrm{~mL}$ de solución buffer (líquido ruminal artificial) y 10 $\mathrm{mL}$ de fluido ruminal obtenido de los ovinos. El líquido ruminal fue filtrado en gasas, manteniendo una temperatura de $39^{\circ} \mathrm{C}$; se realizaron 4 repeticiones. Las UF se introdujeron a un baño de agua a $39^{\circ} \mathrm{C}$ y se inició el registro de la producción de gas. La presión de gas fue determinada en $\mathrm{ml}$ de gas por cada $200 \mathrm{mg}$ de MS.

Determinación de ácidos grasos volátiles (AGV)

La determinación se realizó con el uso de un cromatógrafo de gases (CG) de la marca Agilent Tecnologies, serie 6890N; empleando una columna polar DB_WAXetr. Las condiciones de trabajo fueron: temperatura después de la inyección $250^{\circ} \mathrm{C}$ a una presión de 12.13 psi con un flujo de $\mathrm{He} 36.5 \mathrm{~mL} / \mathrm{min}$. Las condiciones para la columna fueron: temperatura inicial $50^{\circ} \mathrm{C}$ de cero a dos minutos, aumentando de $10^{\circ} \mathrm{C}$ en $10^{\circ} \mathrm{C}$ hasta llegar a $250^{\circ} \mathrm{C}$, manteniendo esta temperatura constante por 5 minutos para luego descender a $50^{\circ} \mathrm{C}$ por dos minutos, con un flujo de He de $1.6 \mathrm{~mL} / \mathrm{min}$ a una presión de 12.13 psi y una velocidad promedio de $25 \mathrm{~cm} / \mathrm{seg}$. Se Utilizó un detector de flama ionizante (FID), a una temperatura de $210^{\circ} \mathrm{C}$, con un flujo de $\mathrm{H}_{2}$ de $40 \mathrm{~mL} / \mathrm{min}$ y un flujo de aire de $450 \mathrm{~mL} / \mathrm{min}$, método descrito por Ewaschuk et al. (2002).

\section{Análisis estadísticos}

El número de semillas por fruto y su peso se evaluó por comparación de medias a través de una prueba $t$ - student, empleando el paquete estadístico PROC MIXED de SAS para evaluar las diferencias estadísticas $(p<0.05)$. La fuente de variación considerada fue el estado de madurez del fruto (verde, maduro y post maduro); mientras que en el análisis proximal las variables consideradas fueron: cenizas, extracto etéreo, proteína, fibra 
cruda, FDN y FDA; considerando como fuentes de variación el germinado y la harina de semilla de guayaba.

\section{RESULTADOS Y DISCUSIÓN}

La cantidad de semillas contabilizadas en las guayabas en estado verde fue de 316 , cantidad mayor que en el estado maduro (306 semillas) y en el estado post maduro (292 semillas); no se encontraron diferencias estadísticas $(p<0.01)$, lo que indica que el número de semillas en las guayabas no se ve afectado por el estado de maduración. Por lo contrario, el peso de las semillas $(\mathrm{g})$ en el estado de maduración verde $(5.1 \mathrm{~g})$, fue mayor a lo observado en el estado post maduro (3.8 g), en este análisis se encontraron diferencias estadísticas $(p<0.01)$ (Tabla 1$)$.

\begin{tabular}{ccc}
\hline Estado de maduración & $\begin{array}{c}\text { Cantidad de semillas } \\
\text { (unidades) }\end{array}$ & Peso $(\mathbf{g})$ \\
\hline Verde & $316.50^{\mathrm{a}}( \pm 65.25)$ & $5.1080^{\mathrm{a}}( \pm 1.25)$ \\
Maduro & $306.50^{\mathrm{a}}( \pm 93.88)$ & $4.7340^{\mathrm{ab}}( \pm 1.01)$ \\
Post maduro & $292.30^{\mathrm{a}}( \pm 112.49)$ & $3.8090^{\mathrm{b}}( \pm 1.21)$
\end{tabular}

Medias con letras distintas en la misma columna difieren estadísticamente $(p<0.01)$; valor entre paréntesis = desviación estándar.

Tabla 1. Valores medios de cantidades de semilla y peso de las guayabas en sus estados de maduración.

En relación con el análisis proximal, los resultados de la germinación de las semillas de guayaba fue de un $92 \%$, en un periodo de cinco semanas; y las plántulas tuvieron una altura máxima $5.7 \mathrm{~cm}$. Así el germinado de guayaba presentó mayor porcentaje de cenizas (1.5\%) y proteína (30.4\%), en comparación con la harina de semilla de guayaba $(p<0.01)$; mientras que el porcentaje de fibra cruda $(46.9 \%)$ y FDN $(22 \%)$ fue mayor en harina de semilla de guayaba, en comparación con el germinado de guayaba; encontrándose diferencias estadísticas $(p<0.01)$. El porcentaje de extracto etéreo fue mayor en la harina de semilla de guayaba, en comparación con el germinado de guayaba; sin embargo no se encontraron diferencias estadísticas $(p<0.01)$ (Tabla 2).

El valor de porcentaje de $40.5 \%$ de extracto etéreo en harina de semilla de guayaba superior al de germinado de guayaba, puede atribuirse a que el embrión requiere una mayor cantidad de reserva de nutrientes y energía, para que la germinación sea favorable; una vez germinada la semilla esta cantidad disminuye (Tabla 2). 


\begin{tabular}{|c|c|c|c|c|c|c|}
\hline & \multirow{2}{*}{$\%$ Cenizas } & \multirow{2}{*}{$\begin{array}{c}\% \text { Extracto } \\
\text { etéreo }\end{array}$} & \multirow{2}{*}{ \% Proteína } & \multirow{2}{*}{$\%$ Fibra cruda } & \multicolumn{2}{|c|}{ A partir de \%FC } \\
\hline & & & & & $\% F D N$ & $\% F D A$ \\
\hline $\begin{array}{l}\text { Germinado de } \\
\text { guayaba }\end{array}$ & $\mathrm{a} 1.517( \pm 0.1)$ & a17.30 ( \pm 2.2$)$ & a30.496 ( \pm 0.73$)$ & a20.189 ( \pm 0.85$)$ & a8.724 ( \pm 0.28$)$ & b7.41 ( \pm 0.28$)$ \\
\hline $\begin{array}{c}\text { Harina de semilla de } \\
\text { guayaba }\end{array}$ & $\mathrm{b}_{1} .096( \pm 0.01)$ & a $40.57( \pm 4.69)$ & b8.788 $( \pm 0.04)$ & b46.960 $( \pm 4.20)$ & b22.053 $( \pm 0.07)$ & 0.00 \\
\hline
\end{tabular}

FC: fibra cruda, FDN: fibra detergente neutro, FDA: fibra detergente ácida. Medias con letras distintas en la misma columna difieren estadísticamente $(p<0.01)$; valor entre paréntesis = desviación estándar.

Tabla 2. Resultado del análisis proximal de la semilla y germinado de guayaba.

El valor en el porcentaje de cenizas de harina de semilla de guayaba es inferior un $0.5 \%$, respecto al valor de germinado de guayaba; las cenizas corresponden a materias minerales y salinas de la muestra, por lo que este porcentaje se encuentra dentro del rango reportado por Vasco et al. (2002).

El incremento de proteína considerablemente mayor en el germinado de guayaba indica su posible uso en la alimentación de rumiantes, lo que no ofrece la harina de semilla; tal y como lo reporta Vasco et al. (2002), en el análisis proximal que realizaron a la harina de semilla mezclada obtenida de la empresa Jugos de Valle.

En la harina de semilla, el porcentaje de fibra cruda fue casi dos veces el valor del porcentaje en el germinado de guayaba, esta disminución se atribuye a la eliminación de la testa que recubre el embrión una vez germinado y constituye el componente principal de la pared vegetal de la planta. Jiménez et al. (2001) indica que la harina de semilla de guayaba además de ser una buena fuente de fibra, es considerada como antioxidante, debido al contenido de polifenoles extraíbles, asociados a la matriz de los componentes de la fibra de esta fruta. La guayaba en comparación con otras frutas especialmente de cáscara, presenta mayores valores de porcentaje de fibra, debido a que este tejido está compuesto por sustancias pécticas, celulosas y hemicelulosas (Wills et al., 1984). Las sustancias pécticas presentes en la guayaba representan la principal fuente de carbohidratos, los cuales son proveedores de energía para los rumiantes, como segunda fuente se consideran la hemicelulosa y la celulosa.

Los valores de porcentaje de FDN en harina de semilla fueron mayores al de germinado de guayaba. La FDN es fibra no digerible por monogástricos, pero es fácilmente digerible por los rumiantes. En los rumiantes actúa como estimulante de la rumia y como generador de saliva, permitiendo un mayor aprovechamiento de los nutrientes presentes en los alimentos. Mientras que la FDA presente solo en el germinado de guayaba (7.4\%), corresponde a la fibra que no es digerible, es decir, en la alimentación de los rumiantes no aporta energía, ya que se considera como material insoluble constituido por celulosa y lignina, por lo que esta fibra actúa solo como satisfactor de alimento en los rumiantes. 
En relación con la técnica de producción de gas, esta se ha utilizado para describir la cinética de fermentación (Menke, 1988) y el valor nutritivo de forrajes (Rymer et al., 2005) principalmente de pajas, granos de cereales, arbustivas y residuos agro-industriales. Los valores nutritivos de la harina de semilla y germinado de guayaba (Tabla 2), fueron analizados con la técnica de digestibilidad in vitro (Van Soest, 1994).

La cinética de fermentación sugiere mayor digestibilidad para el germinado de guayaba ( $40 \mathrm{~mL}$ de gas), cuya producción es mayor a las $48 \mathrm{~h}$ con respecto a la harina de semilla (Figura I). Es importante mencionar que la cantidad de gas producida por gramo de materia seca o de materia orgánica desaparecida, puede ser calculada si las pérdidas de sustrato son cuantificadas, ya sea a diferentes intervalos de tiempo o al final de la fermentación.

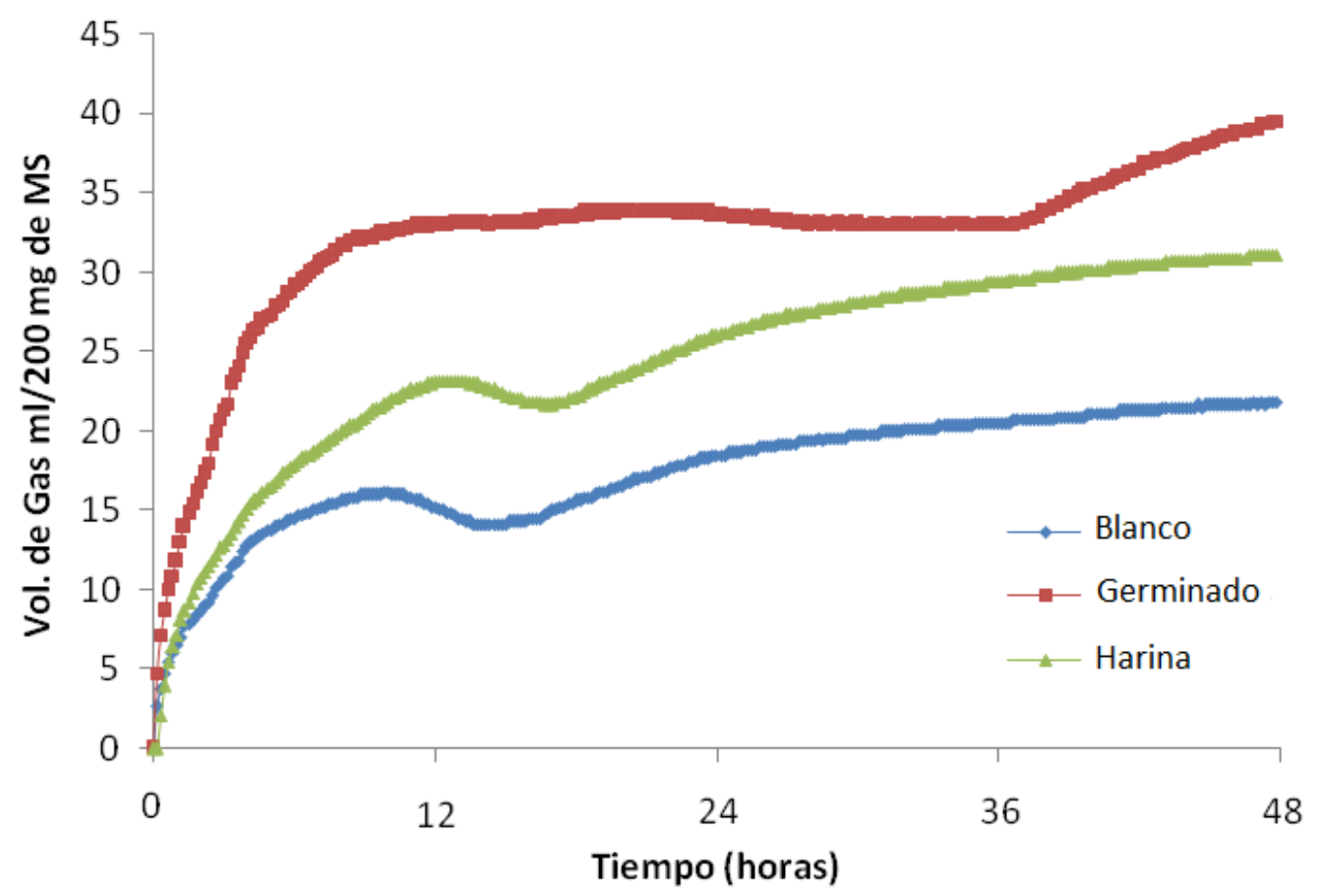

Figura I. Producción de gas in vitro a las 48 horas de incubación de harina de semilla y germinado de guayaba.

La concentración de AGV (ácido acético, ácido propiónico y ácido butírico), el porcentaje de materia seca y el pH de harina y germinado de guayaba se muestran en la Tabla 3 . EI germinado de guayaba presentó la mayor concentración de ácido acético, ácido propiónico y ácido butírico. Las concentraciones de ácido acético y butírico presentaron diferencias significativas $(p<0.01)$. Mientras que la harina de semilla presentó mayores valores en porcentaje de materia seca (diferencias significativas $\mathrm{p}<0.01$ ) y $\mathrm{pH}$ (en este caso las diferencias no fueron significativas, $p<0.01$ ). 
Con base en estos resultados se puede inferir que la harina de semilla de guayaba tiene menores propiedades de digestibilidad comparada con el germinado de guayaba.

\begin{tabular}{|c|c|c|c|c|c|}
\hline & $\begin{array}{l}\text { Ácido acético } \\
\text { (mmol/L) }\end{array}$ & $\begin{array}{l}\text { Ácido propiónico } \\
\text { (mmol/L) }\end{array}$ & $\begin{array}{l}\text { Ácido butírico } \\
\text { (mmol/L) }\end{array}$ & $\%$ Materia seca & $\mathrm{pH}$ \\
\hline Harina de semilla & a21.58 ( \pm 2.12$)$ & a7.94 $( \pm 0.14)$ & a $44.90( \pm 1.41)$ & а39.92( \pm 1.34$)$ & a7.2( \pm 0.28$)$ \\
\hline $\begin{array}{c}\text { Germinado de } \\
\text { guayaba }\end{array}$ & a25.24( \pm 0.34$)$ & $\mathrm{a} 11.26( \pm 0.37)$ & ${ }^{b} 55.8( \pm 1.13)$ & b35.39( \pm 0.49$)$ & a $7.1( \pm 0.21)$ \\
\hline
\end{tabular}

Medias con letras distintas en la misma columna difieren estadísticamente $(p<0.01)$; valor entre paréntesis = desviación estándar.

Tabla 3. Resultados obtenidos de la digestibilidad de harina de semilla y germinado de guayaba.

La cantidad de ácido acético $(25.2 \mathrm{mmol} / \mathrm{L})$ y de ácido propiónico $(11.26 \mathrm{mmol} / \mathrm{L})$ en germinado, fueron similares a los obtenidos en la harina de semilla con resultados de ácido acético (21.58 $\mathrm{mmol} / \mathrm{L})$ y ácido propiónico $(7.94 \mathrm{mmol} / \mathrm{L})$; indican que probablemente si se utilizara la harina de semilla (como fuente de FDN y extracto etéreo) y el germinado de guayaba (como fuente de proteína) como complemento uno de otro, o como complemento en un alimento, se podría formular un alimento "ideal" para los rumiantes y aumentar los AGV generados.

\section{CONCLUSIÓN}

La harina de semilla de guayaba resultó con mayor concentración de grasa y FDN, por lo que puede ser utilizada como complemento en la dieta de los rumiantes. Con respecto al germinado, se descubrió que es una importante fuente de proteína y se puede utilizar como alimento; es decir, tanto la harina como el germinado de guayaba tienen potencial para cubrir los requisitos de mantenimiento de los rumiantes.

\section{LITERATURA CITADA}

AOAC (Association of Official Analytical Chemists). Official methods of analysis (16th ed.). Arlington, VA. USA: Association of Analytical Chemists. 1995.

BEHARKA AA, Nagaraja TG, Morrill JL, Kennedy GA, Klemm RD. Effects of form of the diet on anatomical, microbial, and fermentative development of the rumen of neonatal calves1. Journal of Dairy Science. 1998. 81(7): 1946-1955. DOI: 10.3168/jds.S00220302(98)75768-6.

BERNARDINO NA, Ortiz MA, Martínez AAL, Dávila OG. Guava seed protein isolate: Functional and nutritional characterization. Journal of Food Biochemistry. 2001. 25(1), 7790. DOI: $10.1111 /$ j.1745-4514.2001.tb00725.x 
EWASCHUK JB, Zello GA, Naylor JM, Brocks RB. Metabolic acidosis: separation methods and biological relevance of organic acids and lactic acid enantiomers. Journal of Chromatography B. 2002. 781(1), 39-56. DOI: 10.1016/S1570-0232(02)00500-7

FRANCE J, Siddons RC, Dhanoa MS. Adaptation of compartmental schemes of interpreting isotope dilution data on volatile fatty acid metabolism in the rumen to the nonsteady state and for single-dose injection. Journal of Theoretical Biology. 1991. 153(2): 247-254. DOI:10.1016/S0022-5193(05)80425-4.

GEORING HK, Van Soest PJ. Forage fiber analyses (apparatus, reagents, procedures, and some applications). ARS-USDA, Washington, DC Agric. Handbook. 1970. 379.

GUPTA GK, Chahal J, Arora D. Psidium guajava Linn.: Current research and future prospects. Journal of Pharmacy Research. 2011. 4(1): 42-46.

JIMÉNEZ EA, Rincón M, Pulido R, Saura CF. Guava fruit (Psidium guajava L) as a new source of antioxidant dietary fiber. Journal of Agricultural and Food Chemistry. 2001. 49(11), 5489-5493. DOI: 10.1021/jf010147p

JOSEPH B, Priya RM. Phytochemical and biopharmaceutical aspects of Psidium guajava (L.) essential oil: a review. Research Journal of Medicinal Plant. 2011. 5(4): 432-442.

KAUFFMAN W. Influence of the composition of the ration and the feeding frequency on $\mathrm{pH}$ regulation in the rumen and on feed intake in ruminants. Livestock Production science. 1976. 3(2): 103-114.

MENKE KH, Steingass $\mathrm{H}$. Estimation of the energetic feed value obtained from chemical analysis and in vitro gas production using rumen fluid. Animal research and development. 1988. 28: 7-55.

MERTENS DR. Predicting intake and digestibility using mathematical models of ruminal function. Journal of Animal Science. 1987. 64(5): 1548-1558.

MEZA N, Bautista D. Morfología de semillas de guayabo (Psidium guajava L.), germinación y emergencia después del remojo en agua. Revista de la Facultad de Agronomía. 2007. 24(01): 265-270.

NRC. Nutrient Requirements of Dary Cattle. 6th ed. National Academy Press. Washington, DC. 2007.

PEREIRA TS, Andrade AD. Germinação de Psidium guajava L. e Passiflora edulis Sims: efeito da temperatura, substrato e morfologia do desenvolvimento pós-seminal. Revista Brasileira de Sementes. 1994. 16(1): 58-62. 
PÉREZ AT, Nápoles L, Concepción O, Trujillo R. Multiplicación in vitro de brotes de guayaba (Psidium guajava L.) var. Enana roja cubana EEA 18-40 obtenidos a partir de $\begin{array}{llll}\text { semillas. } & \text { Cultivos } 2002 . & \text { 23(3): } & \text { 57-61. }\end{array}$ http://www.redalyc.org/pdf/1932/193218120008.pdf

DEL PILAR PIM, Fischer G, Corredor G. Determinación de los estados de madurez del fruto de la gulupa (Passiflora edulis Sims.). Agronomía Colombiana. 2007. 25(1): 83-95

RISHIKA D, Sharma R. An update of pharmacological activity of Psidium guajava in the management of various disorders. International Journal of Pharmaceutical Sciences and Research. 2012. 3(10): 3577-3584. DOI: 10.13040/IJPSR.0975-8232.3 (10).3577-84

RYMER C, Huntington JA, Williams BA, Givens DI. In vitro cumulative gas production techniques: History, methodological considerations and challenges. Animal Feed Science and Technology. 2005. 123: 9-30. DOI: 10.1016/j.anifeedsci.2005.04.055

SAGARPA (Secretaria de Agricultura, Ganadería, Desarrollo Rural, Pesca y Alimentación). Atlas agroalimentario 2015, México. 2015. 84-85.

SANDA KA, Grema HA, Geidam YA, Bukar KYM. Pharmacological aspects of Psidium guajava: An update. International Journal of Pharmacology. 2011. 7:316-24. DOI: 10.3923/ijp.2011.316.324

SIAP (Servicio de Información Agroalimentaria y Pesquera). Información digital. www.siap.gob.mx. México. 2012.

SUTTON JD. Digestion and end product formation in the rumen from production rations. In: Ruckebusch $\mathrm{Y}$ and Thivend $\mathrm{P}$ (Editors) Digestive physiology and metabolism in ruminants (ed. Y. Ruckebusch and P. Thivend). MTP Press, Lancaster. 1980. 271-290. DOI: 10.1007/978-94-011-8067-2_13

VASCO MNL, Guevara RI, Acero GMG, Toro VJF. Chemical composition of seeds and oil of guava (Psidium guajava L.). Scientiae Naturae. 2002. 4(2): 25-32.

WILLAN RL. Guía para la manipulación de semillas forestales, estudio con especial referencia a los trópicos. FAO Montes. 1991. 20(2): 502.

WILLS RH, Lee H, McGlasson B, Graham D. Fisiología y Manipulación de Frutas y Hortalizas Postrecolección. Editorial Acribia, Zaragoza. 1984. 192. 